proportions meeting cut-off for HT or PreHT/'Elevated BP' (EBP) based on British vs AAP/ESH reference definitions.

Methods Between March 2019 and March 2020, 67 CYP who attended routine clinician-led appointments for ADHD medication review had their BP and Pulse measured with electronic sphygmomanometers. The equipment was regularly calibrated and clinicians followed standard procedures for checking BP and Pulse.

Results Of the 67 CYP, 85\% were males, and the average age of the cohort was 12 years. Based on the British definition, $12(18 \%)$ were recorded as PreHT while $7 \%$ met the criteria for HT. The corresponding proportion of CYP meeting the AAP/ESH definitions for EBP and HT were 15\% and 7.5\% respectively. the CYP with HT or PreHT were monitored with non-clinic based BP measurements at home or by the GP and all returned to normal in the following eight months.

Conclusions The proportions of CYP classified by the British or AAP/ESH reference as having HT or PreHT were different albeit with small margins. However these small differences in proportion could have population-level implications if mapped onto the full cohort of CYP with ADHD attending similar medication reviews in the UK. Also, the differences could lead to frontline clinicians using different thresholds for triggering remedial actions in CYP with suspected HT such as medication dose reduction or paediatric cardiology referral. Therefore, we recommend further review of these different reference points to avoid confusion among frontline clinicians. One potential reason for the differences is that the British reference does not consider the CYP's height. Perhaps, including height in the algorithm that determines all BP reference centiles for CYP would provide closer results.

\section{LONGITUDINAL STUDY OF BLOOD PRESSURE AMONG CHILDREN AND ADOLESCENTS WITH ADHD SUGGESTS HIGH LEVELS OF STRESS AND ANXIETY DURING THE COVID-19 PANDEMIC LOCKDOWN}

Michael Ogundele. UK

\subsection{6/bmipo-2021-RCPCH.174}

Background The whole world is experiencing an unprecedented period of severe social disruption and economic downturn due to global spread of a rare new strain of Severe Acute Respiratory Syndrome (SARS) virus called Coronavirus. Children and Young people (CYP) are less likely to have severe symptoms from acute COVID-19 infections, but they are more prone to possible several adverse mental health outcomes. Social isolation (eg, marital status, number of close friends and relatives, religious or other group affiliations) is known to be a major cause of stress and risk factor for cardiovascular disease including elevated Blood Pressure (BP) and Heart rates (HR).

Objectives This study aimed to analyse the effect of the UK pandemic national lockdown on the cardiovascular health (CVH) of CYP with ADHD regularly followed up in a North West England Community Child Health Clinic. We compared the BP and HR measurements for the CYP before and after the COVID-19 pandemic lockdown (CPL) to assess its effects on their $\mathrm{CVH}$.
Methods The CVH of 57 CYP with ADHD was prospectively studied during their clinic reviews, before and after the official commencement of the national UK lockdown on the 26th March 2020. Pre-Hypertension (PreHT)/Elevate BP (EBP) and Hypertension (HT) were defined using both the Great Britain reference charts (between 91st and 98th centile or above 98th centile for age) and the US-based recommendations (between 90th and 95th centile or above 95 percentile for gender, age and height) respectively. The centiles for HR measurements were derived from the published reference data from existing international studies of normal children. Student paired T-test was used for comparison of pre/post CPL BP/HR data.

Results The clinical characteristics of CYP seen before and after the CPL were similar in terms of average age, number of co-morbidities, use of medications and gender distribution.

The HR, diastolic and systolic BP of all the CYP with ADHD significantly increased during the CPL $(p<0.001)$. There was two- to four-fold increase in the number of the CYP with HT, PreHT/EBP measured after the CPL (figure 1). Diastolic BP elevation accounted for $89 \%$ to $100 \%$ of the abnormal BP measurements. This increase in measured $\mathrm{BP}$ and HR could not be attributed to any prescribed ADHD medications as the patients had been on the same medications and unchanged dosage for an average of more than 16 months, all within the lower range of normal doses.

There was statistically significant correlation between the elevated systolic and diastolic BP, and the number of weeks from the inception of the CPL.

All the CYP with elevated BP are being closely monitored in the clinic and at home. The elevated BP measurements have gradually resolved as the lockdown was eased and no long-term negative consequences have been observed.

Conclusions We conclude that the CPL was associated with transient and reversible increase in the BP and HR of CYP with background diagnosis of ADHD and other co-morbid neurodevelopmental disorders. This is probably an indication of the psychological distress associated with the pandemic affecting CYP, as also previously described for adults.

\section{BEFORE SCALING-UP, SPEAK WITH THE STAKEHOLDERS: LESSONS FROM A PRETERM PROJECT}

Josephine Agyeman-Duah, Anita Appiah, Jacqueline Asibey, Ernest Asiedu, Stephen Kennedy, Jose Villar. UK

\subsection{6/bmjpo-2021-RCPCH.175}

Background In 2019, the [1]National Catholic Health Service (NCHS) initiated the INTERPRACTICE-21st Study in Ghana: implementation of the [2]INTERGROWTH-21st (IG21) Preterm Postnatal Growth Standards and Feeding Protocols within its hospitals.

Implementation included

1. Monitoring the growth of preterm babies with the IG21 standards, rather than the traditional growth charts for term babies.

2. Training health professionals involved with neonatal care to use the IG21 standards and evidence-based, feeding protocols (with their focus on the use of breast milk). 
Abstract 316 Table 1 Emerging themes from interviews

\begin{tabular}{|c|c|c|}
\hline Strengths and enabling factors & Limitations/challenges & Recommendations for improvement \\
\hline Better monitoring of preterm baby growth & Continuous on-site supportive monitoring & Staff motivation through supportive supervision \\
\hline Involvement of paediatricians as local leads & Data sharing & $\begin{array}{l}\text { Data management: } \\
\text { - Data quality improvement including providing feedback to facilities } \\
\text { - Involving nurses to coordinate data monitoring } \\
\text { - Enrolling health information officers to support doctors to manage data } \\
\text { - Publication is an incentive for doctors } \\
\text { - A harmonised records book to include preterm growth charts and } \\
\text { indicators }\end{array}$ \\
\hline Supportive supervision & Impact of Covid-19 & Embedding sustainable funding model before scale-up \\
\hline Communication & $\begin{array}{l}\text { Attitudes and priorities: more focus on mother than preterm } \\
\text { baby }\end{array}$ & Active involvement of parents, caregivers and community \\
\hline Management support & & Identifying strategic alliances for local and global partnerships \\
\hline \multicolumn{3}{|l|}{$\begin{array}{l}\text { Quality Improvement as a service-wide } \\
\text { culture }\end{array}$} \\
\hline \multicolumn{3}{|l|}{ Staff commitment } \\
\hline Parent involvement & & \\
\hline
\end{tabular}

\section{Partner consultations.}

We present the results of the qualitative component of an evaluation study, conducted after the 15 month pilot phase, to inform future [3]spread and [4]scale-up of interventions.

[1] https://www.nchs.org.gh

[2] https://intergrowth21.tghn.org/interpractice-21st/

[3] Spread definition: https://qi.elft.nhs.uk/collection/scaleup-and-spread/

[4] Scale-up definition: https://qi.elft.nhs.uk/collection/scaleup-and-spread/

Objectives

- To describe stakeholder views and perspectives regarding the introduction of a novel public health intervention aimed at improving care for preterm babies in a low-resource setting.

- To compile lessons learned for national scale-up of preterm interventions.

Methods A questionnaire was developed based on the literature relating to implementation science. In-depth interviews were conducted with eight key stakeholders who were purposively sampled because of their prior knowledge of the INTERPRACTICE-21st Study and/or preterm care in Ghana. Eligible stakeholders were invited via email to participate. Interviewees included officials at the Ministry of Health and NCHS, and other stakeholders in the field of paediatrics and public health.

Due to COVID-19 restrictions, interviews were conducted virtually (via a video call). Verbal consent was obtained prior to the interviews. Recorded audio files were transcribed and thematic content analysis was performed using [5]Pope's Approach to extract emerging themes from the interviews. The Oxford Tropical Research Ethics Committee approved the study (no. 536-20).

[5] https://www.bmj.com/content/320/7227/114

Results Emerging themes from the interviews are presented in table 1 below:

Conclusions

- Stakeholder responses show positive feedback to the pilot phase of the project.
- The study emphasises the need to seek the views of stakeholders in a pilot phase before embarking on nationwide scale-up of a preterm intervention in a low-resource setting.

\section{COMMON DIAGNOSES AMONG PEDIATRIC ATTENDANCES AT EMERGENCY DEPARTMENTS}

Shuen Yin Celine Yoong, Shuen Yin Celine Yoong, Yong-Kwang Gene Ong, ShuLing Chong, Peck Har Ang, Nur Diana Bte Zakaria. Singapore

\subsection{6/bmjpo-2021-RCPCH.176}

Background Pediatric patients present to Emergency Departments (EDs) with a variety of medical conditions. An appreciation of the common presenting conditions can aid EDs in the provision of pediatric emergency care.

Objectives We aimed to determine the common pediatric diagnoses seen, and how they differed between pediatric-only and general EDs.

Methods A retrospective review of medical records was performed for patients less than 16 years old at a pediatric ED and three general EDs from 1 January to 31 December 2018. Information including patient demographics, triage category, case type and diagnoses were collected.

Results There were 161,375 pediatric attendances, of which $155,563(96.4 \%)$ were seen at the pediatric ED. Non-traumatic conditions were most prevalent in both pediatric $(\mathrm{N}=128,415,82.5 \%)$ and general EDs $(\mathrm{N}=3,506,60.3 \%)$. Trauma related conditions were more commonly seen in general EDs $(\mathrm{N}=2,306,39.7 \%)$ compared to the pediatric ED $(\mathrm{N}=27,148,17.5 \% ; \mathrm{p}<0.01)$. Across all EDs, fracture, wound and head injury were the three most common trauma related diagnoses, while upper respiratory tract infection, unspecified fever and gastroenteritis were the three most common nontrauma related diagnoses. There was a greater proportion of emergent (P1) cases seen in general EDs $(\mathrm{N}=277,4.8 \%)$ than the pediatric $\mathrm{ED}(\mathrm{N}=3,821,2.5 \% ; \mathrm{p}<0.01)$. Respiratory conditions including bronchiolitis, asthma and bronchitis were the most common emergent (P1) diagnoses. 